\title{
Platform Architecture for Internet Service Delivery and Management
}

\author{
Gaëtan Vanet, Motohiro Suzuki, Tôru Egashira, and Yoshiaki Kiriha \\ Computer \& Communication Media Research \\ NEC Corporation \\ 1-1, Miyazaki 4-Chome, Miyamae-Ku, \\ Kawasaki 216-8555, Japan \\ \{vanet, motohiro,egashira,kiriha\}@ccm.cl.nec.co.jp
}

\begin{abstract}
Through the current explosion of the Internet, service consumers expect advanced Internet services become more customizable and easy to use. So, Service Providers must be able to deploy and manage such services, reducing the time to market of its services while drastically improving the Service Quality. However, the current Internet technology is lacking when it comes to developing and deploying new services, integrating together service offerings. This paper proposes a platform architecture for Internet Service delivering and management. We also introduce the concept of "basic service component" as an extension of the notion of building block defined by the TeleManagement Forum and as a way to build business services in a flexible manner. Our proposal makes full use of the Telecommunications Information Networking Architecture (TINA) Business Model with some adaptations to fit with our component-based approach.

Keywords. Basic Service Component, Building Block, Composite Internet Service, TINA Business Model.
\end{abstract}

\section{Introduction}

Through the current explosion of the IP technology, service customers expect the Internet to become a multiservice worldwide platform, supporting a full range of advanced IP services including video conferencing, application rental or network based training service. Besides, data services are expected to grow to around 70 percent of the network bandwidth use within the next 5 years. However, this multi-billion market has significant implications for Service Providers.

Service Providers have to comply with different requirements of the market: as customers have now the choice between different providers, the competitive context of the Telecommunication environment requires the Service Provider to reduce the time to market of its services. Moreover, service customers now expect advanced IP services become more intelligent and more customer-specific, e.g. adaptable to each individual requirements while drastically improving the Service Quality. This service customization entails different service compositions and service presentations, proposing, for instance, different types and contents of user interface to customers. Furthermore, the behavior of customers has drastically changed and the service delivery must fit with the variety of customer service access capabilities which can include laptops, PDAs and mobile phones. Finally, the service fulfillment process must be done dynamically, according to the requirements of the users and the conditions of the network. The finality of this evolution is to make advanced IP services "consumable", e.g. pay-per-use, and easy to use like the telephone today.

\footnotetext{
A. Ambler, S.B.Calo, and G. Kar (Eds.): DSOM 2000, LNCS 1960, pp. 95 - 106, 2000.

(C) Springer-Verlag Berlin Heidelberg 2000
} 
The main challenge for service providers is the management of such new services which may rely on mechanisms like policy-based management, Service Level Agreement (SLA) management, Customer Network Management (CNM), and automatic remote network configuration and monitoring. In this context, the current Internet technologies are lacking when it comes to developing and deploying new services, integrating together service offerings. Even if this is a complicated challenge, the combination of technologies like Active Networking, Workflow Engine and Application Components can provide a suitable solution to this challenge.

The following section introduces the evolution of the Telecommunication Business model and discusses why the active network, the application components and the workflow engine technologies can constitute a suitable combination to deploy and manage advanced IP services. Section 3 introduces the concept of basic service components and IP service modularity. Then, we presents our proposal of platform architecture in section 4 . The service fulfillment and assurance processes are detailed in section 5. Our implementation relying over the NEC front-end middleware is introduced in section 6. Section 7 concludes this paper and presents topics for further research.

\section{Evolution of the Telecommunication Business Model}

As we aforementioned, business models of the past are not suitable anymore and must be updated to fit with the evolution of the Telecommunication Business Environment. Indeed, new business models try to end up with the monolithic system wherein the network provider is connectivity provider, content provider and retailer at the same time. Figure 1 represents the Business Model defined by the Telecommunications Information Networking Architecture Consortium (TINA-C). For further details about the role of each business role and business relationship, the reader should refer to [10].

In the current and popular model of the Internet environment, four actors have unique roles in supplying or consuming advanced IP services: the service consumer, the Application Service Provider (ASP), the Network Service Provider (NSP) and the Service Portal. The ASP plays the role of the 3rd Party Service Provider defined in the TINA-C model. It focuses its activity on providing a growing range of advanced IP services including application rental or video conferencing. The ASP can be either a service logic provider or a content provider or both. It develops the code necessary for the implementation of these IP services that will be deployed within the network. The NSP, the connectivity provider, sells added-value services analogous to the multitude of enhanced voice services that are offered to customers along with basic voice transport. We believe that the NSP must provide customers with services going beyond the classical network services in order to survive in this new telecom environment. Finally, the Service Portal plays both the role of service retailer and broker, providing a portal to individual and corporate customers. Yahoo or Altavista are good examples of popular Service Portals. According to this Business Context, the dynamic insertion of code, for both the service logic and the service management, is necessary to provide customers with enough service flexibility. Furthermore, we must increase the use of functional service components to enable advanced IP services to be more customizable and code more easily re-usable. 


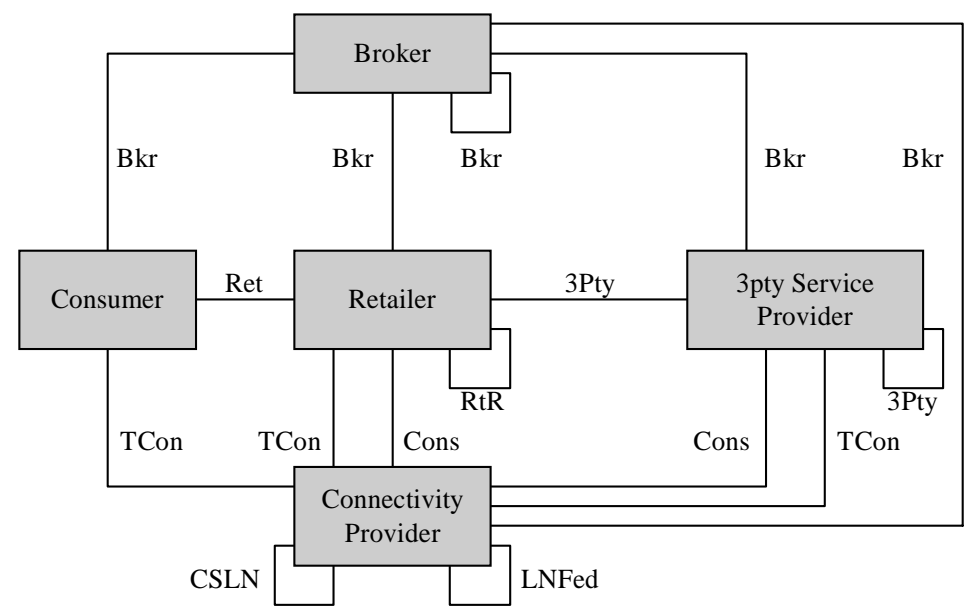

Figure 1. TINA Business Model Relationships Types.

The Active Network technology [6,13] enables the insertion of new services in the network. As Juhola and al. mentioned [4], Active Networking can improve the ability of network providers to penetrate the lucrative Internet-based markets by bringing the following benefits: a) inherently mobility of users, b) more available services based on library of service components, c) additional flexibility to facilitate fast service introduction and enhancements for complex services. We also anticipate that $3 \mathrm{rd}$ party development of value added services will arise, providing customers with an increased range of application level services in response to their various needs. In addition, the work of the TeleManagement Forum (TM Forum) to provide guidelines to design application architectures using the concept of Building Blocks [11] provides services with better modularity. This application components-based approach can reduce the time-to-market while also proposing re-usable code and customizable services. In other respects, the Workflow technology can link these service blocks together in a dynamic and flexible manner. The aim of this paper is to propose a platform architecture to deploy and manage Internet services. In this architecture, Internet services are composed of service components and the workflow engine is presented as one technology to link these components together. Our proposal for a new platform architecture for delivering and managing component-based IP services is compliant with the efforts of TINA-C and TM Forum to define truly open architectures.

\section{The Service Modularity}

As we mentioned in the introduction, IP services must become more customizable, rather viewed as an integration of basic service components than a static and unified service, common to all customers as it is the case today $[1,5,14]$. In fact, the concept of component should be rather considered as an evolution of the object-oriented development methodologies to enable better system integration than its direct competitor. The CORBA Components [9] and Enterprise JavaBeans [3] technologies are the main actors driving this evolution towards component based systems. In these emerging standards, a component is a reusable program block that provides a business logic and can be combined with others in a distributed manner to build an application. 


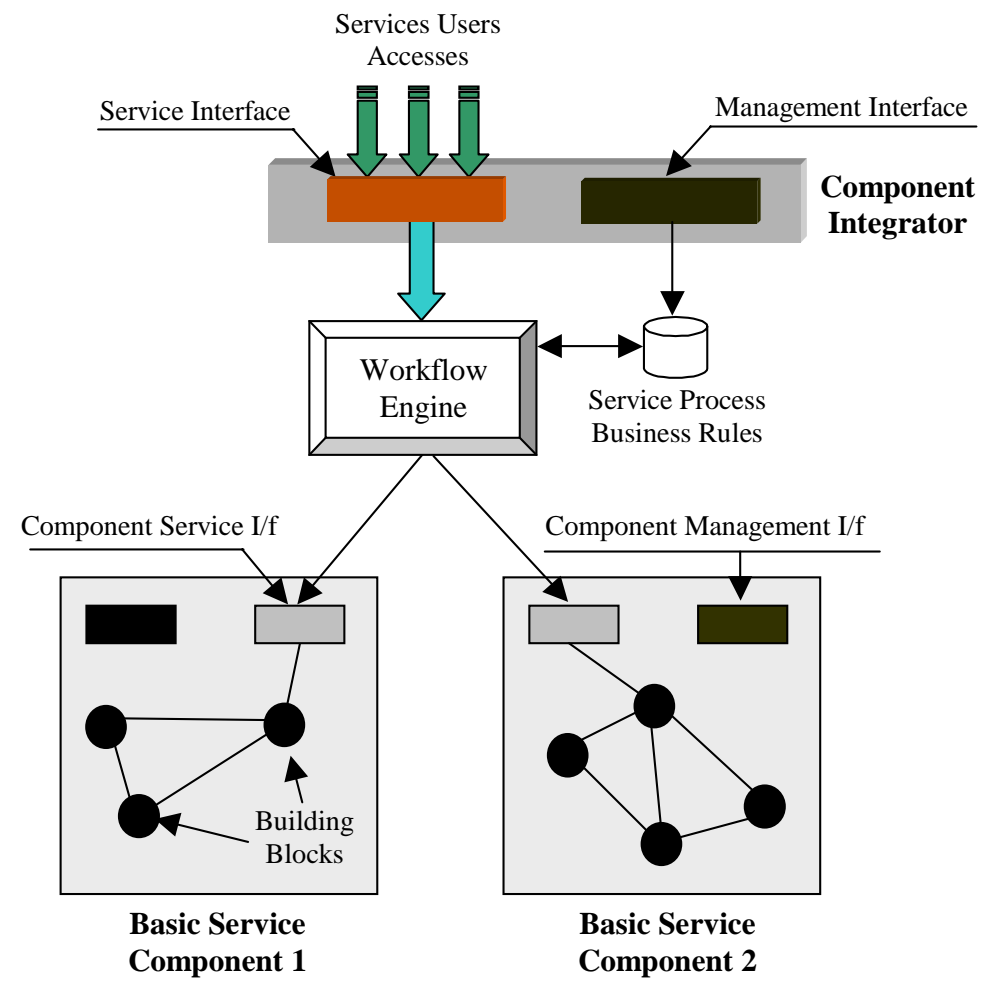

Figure 2. Model of Component-Based IP Service.

In our proposal, we indeed make the distinction between the views of the service that the customer and the provider may respectively have. From the customer's point of view, the service is the business product he pays for and can be confined to the role of Service Access Point (SAP). For the service provider, the service also includes all the service components which must be implemented to provide customers with this business product. In this document, we called them "basic service component". Each service component is re-usable and sharable between different business services.

Figure 2 represents the model of a component-based IP service. Thus, a business service is a composition of basic service components. The integration of these service components is done by the Workflow Engine. The Workflow Engine schedules the flow of messages between the different basic service components. Some rules are associated with this workflow system to detail the flow of data between the different basic components providing the business service. Currently, different research projects $[7,14]$ are investigating the use of workflow system to enable management application integration. However, not so many efforts are done towards the integration of service components. In our proposal, the service users access the service through the Component Integrator (CI). The Component Integrator is composed of a service interface and a management interface. The service interface is intended for service customers to use the service. It provides customers with the list of all the methods related to the specific 
service. On the other hand, the management interface enables the manager to set-up, supervise and control the service. For instance, he can update the rules defining the workflow process. The Component Integrator also contains service meta-data defining the type and the location of the basic service components which must be assembled to build up the corresponding business service.

A Basic Service Component is also composed of two different interfaces: a management and a service interface. The component management interface enables the component manager to start/stop the component, get the number of customers connected, the transaction rate or the computing resource usage. On the other hand, the component service interface lists the capabilities related to the specific service logic provided by the service component. In the case of a basic service component proposing a billing service, the service interface must include capabilities to configure the component, manage a client billing profile, and so on. To make an analogy with the building block concept elaborated by the TM Forum in the Telecom Integration Map document [11], the basic service components are both composed of different building blocks with functionalities that may belong to different computing tiers. They can be implemented as a process component based on the CORBA Components specification [9].

\section{Platform Architecture for Internet Services}

As we explained in the previous section, this is very promising to design a service platform architecture that considers a service as a composition of basic and reusable service components. However, the deployment and the management of such services is a difficult task for Service Providers. This section details our proposal of platform architecture for delivering and managing these composite Internet services.

\subsection{Overview}

The authors of the FlowThru project [7] has proposed a mapping of the TM Forum Business Processes onto TINA Business Roles. In their mapping, the operational processes defined by the TM Forum are assigned to each business role of the TINA Business Model. Their model was defined to be applied to the Telecommunication Environment but can be modified to fit with our component-based Internet service approach.

So, we propose in this paper a new mapping to fit with the requirements of the IP services environment and with our composed-based services approach. This mapping is depicted in figure 3 . We kept the same business roles as defined by the TINA Consortium. However, we modified the business processes applied to each business role. First, we consider two types of 3rd party service provider: the first one is a Basic Service Component Provider which provides the service logic while the second one is a Content Provider, focusing its activity only on the content of the service. However, the TINA Business Model defines the business role of Connectivity Provider that we do not have in figure 3. In fact, we are considering one more hierarchical level and the connectivity issue is masked. On the other hand, we introduced new business processes into the 3rd party service provider role to manage the basic service components (BSC) or the content elements. The broker, consumer and retailer business roles are as defined by the TINA Consortium. Actually, the IP services world requires the consideration of the computational aspect of the service components, aspect which is cur- 


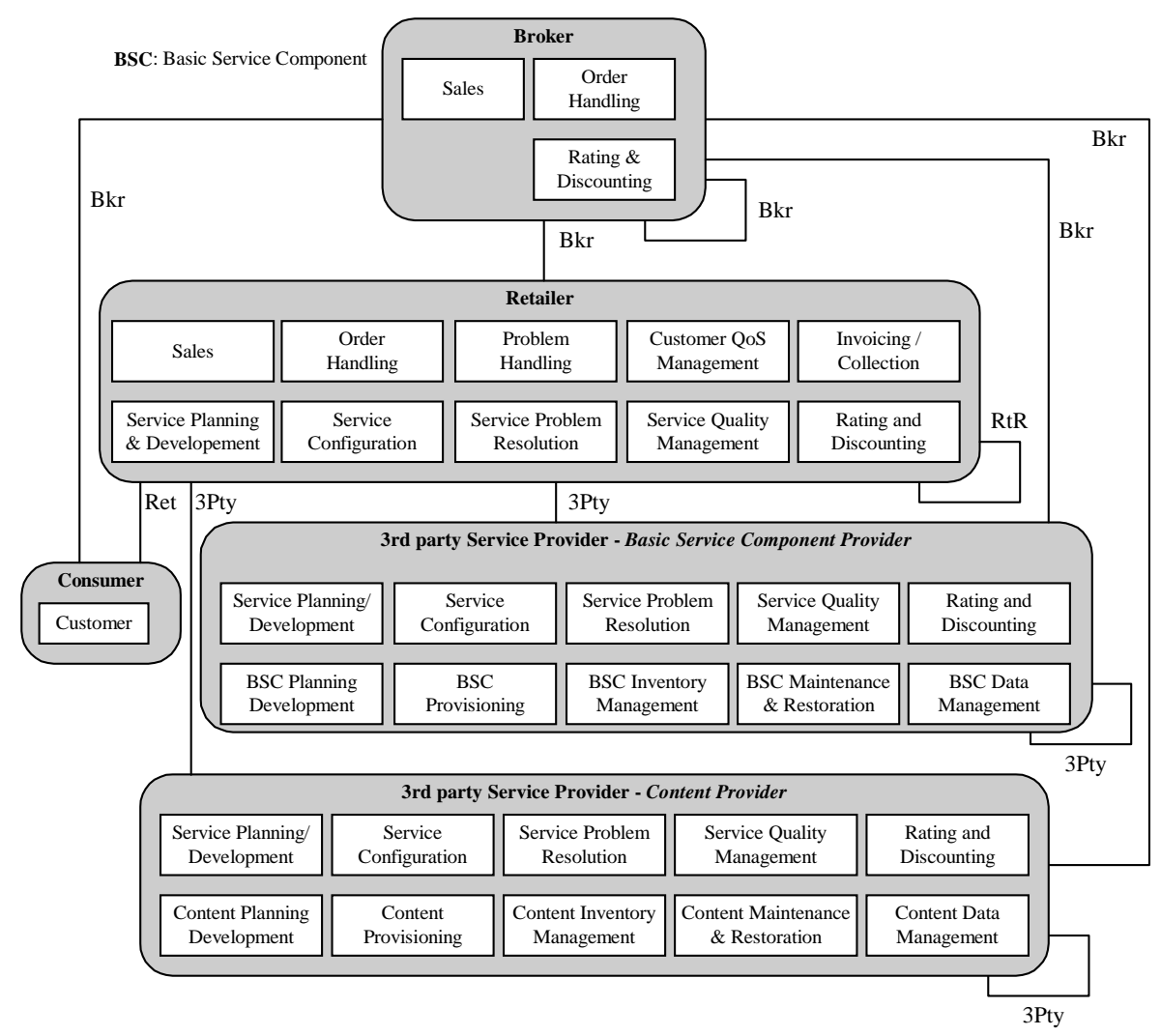

Figure 3. Mapping of the TM Forum Business Processes onto TINA Business Roles Applied to the Component-Based IP Services Scenario.

rently missing in the TINA Business Model. This feature has been taken into consideration for the elaboration of the model depicted in figure 3.

\subsection{Business Roles Description}

The previous section introduced the overview of our service platform proposal. As our proposal focuses on the deployment and management of component-based services, we are going to explain into further details the functions of the Retailer and 3rd Party Service Provider - Basic Service Component Provider presented in figure 3. In this section, we define components composed of one or several Business Processes.

\subsubsection{The Retailer}

As a Service Contractor, the retailer guarantees the service by providing the necessary Service Access Points and fulfilling the conditions defined in the SLA, signed with the service consumer. It also collects the accounting information for the purpose of billing. It manages the service user profiles, processing policies and achieves the authorization prior to service usage. Indeed, the service retailer is responsible for the business processes specified in figure 3. These have been defined by the TM Forum [12] but we 
explain in this section their function considering our composite Internet services approach.

The Sales process component comprises the functions necessary for the interactions with the customer, the sale of the services and the translation of customer requests into right actions. The classical HTTP might be the suitable support for the interactions with clients. It also achieves the management of the Service Access Points spanned throughout the network and maintains the repository of all the services which can be provided to clients.

The Order Handling and Service Configuration process together comprise all the management functions needed in order to define service offerings, administer customers and users, and manage the details of service provisioning. In our proposal, these processes span the service and basic service component layer. According to the service chosen by the customer, it defines the list of basic service components which must be implemented to provide this business service and contacts the right 3rd party service providers to ask them to deploy these components.

The Customer QoS Management process checks if the SLA signed with the service consumers are respected. In our case, this process supervises the end-to-end service, at the level of the Component Integrator. It does not check the QoS at the basic service component level. It manages all the data related to clients including SLA requirements, the list of services he can access and the corresponding QoS. It monitors, manages and reports the quality of service. The Service Quality Management process monitors the service at the service class level. Indeed, this process supervises the Quality of Service and if the service levels are not met, it must achieve some reconfiguration. In our composite service approach, the retailer may decide to contact 3rd party service providers to deploy new service components.

The Problem Handling process achieves the interactions with the service client concerning the service problems. If the customer notifies a SLA violation, this process contacts the Service Problem Resolution process. This latter first defines the basic service components composing this service. Then, it contacts the different basic service providers to notify them about this problem. These must then find the solution to solve this service trouble.

The Service Planning \& Development, the Invoicing/Collection and the Rating and Discounting process are similar as those defined in the TM Forum Telecom Operations Map document [12]. The Invoicing/Collection process manages the billing while the Rating and Discounting process applies the basic rating rules and the discounting policies if the Service Level Agreements were not met.

\subsubsection{Basic Service Component Provider}

The Basic Service Component Provider business role is a 3rd party service provider. Its main function is the provisioning and management of the basic service components rent to service retailers. Indeed, the Basic Service Component Provider is responsible for the business processes specified in figure 3. These have been defined by the TM Forum [12] but we explain in this section their function considering our composite Internet services approach.

The Basic Service Component Provisioning process manages the provisioning of the basic service components required by service retailers. This deployment of basic service components in the network is done according to the requests of retailers or $3 \mathrm{rd}$ party service providers. The Basic Service Component Inventory Management is in- 
volved in the code management of the service components. The basic service components repository can be distributed throughout the network and the code of these components can be stored in code servers. The Basic Service Component Planning/Development process deals with the development and the acceptance of new service components. It can also contact other Basic Service Component Providers to provide better offers to service retailers.

The Basic Service Component Maintenance \& Restoration process monitors the service components installed and running. If it detects abnormalities, it can re-act based on rules. For instance, it replaces service components or deploys new ones. The Basic Service Component Data Management process gets a collection of usage data and events necessary for the purpose of service components performance and service analysis. This process may gather information from agents monitoring the "health" of basic service components or directly through events emitted by the basic service components themselves.

The 3rd party service provider can have business relationship with other Basic Service Component Provider to provide service retailers with a more complete offer. In this case, the Service Configuration, the Service Problem Resolution, the Service Quality Management, the Service Planning/Development and the Rating and Discounting process have the same function as specified above for the service retailer but related to these "outsourced" service components.

\section{Internet Service Management}

The Service Management includes the installation, running, supervision and removal of a service in a networking infrastructure. The TM Forum Telecom Operations Map [12] has defined three major steps in the "Flow-Through" process followed by a service provider to provide clients with services: fulfillment, assurance and billing. The fulfillment and the assurance steps are detailed in this section.

\subsection{Service Fulfillment}

The Service Fulfillment process is responsible for timely delivering what the customer ordered. The design, deployment and activation of advanced IP services require multiple steps. Firstly, the 3rd party service providers contact the service retailers to indicate the basic service components they can provide along with the corresponding SLA. Then, the service consumer connects the network via classical dial-up access and connects the service retailer. This latter defines the Service Home Page (SHP) specific to this customer. This SHP can be considered as a user interface, Web page or Java applet, listing all the services the consumer may access. Afterward, the client chooses the service he wants to access along with the required SLA. The Order Handling process of the service retailer checks the rights and the credit of this customer and creates his session account. After that, the Service Configuration process defines the list of the basic service components which must be implemented to provide the service. Next, the service retailer contacts the 3rd party service providers which sell these components and specifies the location where these components must be installed.

The Basic Service Component Provisioning process of the 3rd party service provider locates the basic service components that are already implemented in the network. If the right components are not already in place, it deploys them. Actually, each basic service component has its own SLA signed between the service retailer and the 


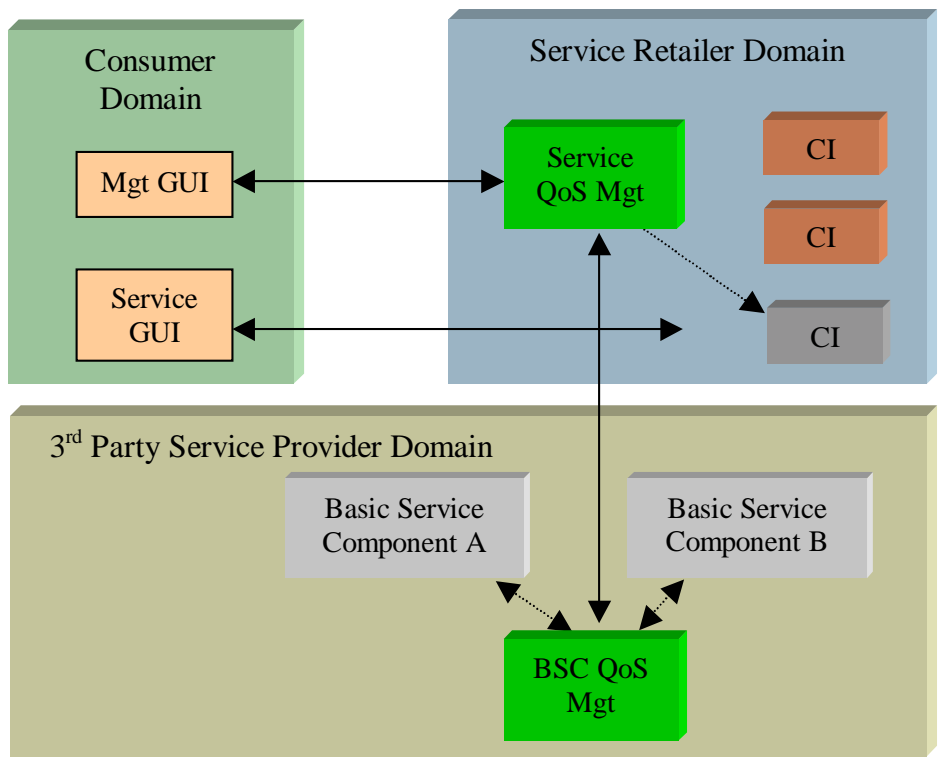

Figure 4. Example of Service Assurance Process.

3rd party service provider. Afterward, the service retailer deploys and activates the service interfaces the service consumer will access to use his required business service. The service profile business rules of the workflow engine must also be updated to take into account this service provisioning phase.

\subsection{Service Assurance}

In the current state of the Telecommunication environment, Service Providers wants to provide their customers with SLAs, stating the obligations defined between the provider and the consumer. The aim of the Service Assurance process is to control that the conditions specified in SLAs are respected and if it is not the case, it must find the source of this "trouble" and correct it. Besides, the Service Assurance will have to apply the service discount policy to the customer according to the level of the "trouble" damage. In order to present the communication between the different entities of our platform architecture for IP services management, we consider the scenario depicted in figure 4 . In this example, a business service is composed of two basic service components, called A and B, provided by a Basic Service Component Provider.

Two GUIs have been defined in the Consumer Domain. The purpose of the Management GUI is to enable the service client to check the Quality of his Service. The Service GUI is the interface provided by the Service the client is accessing. The service retailer plays the role of Quality and Accounting Manager concerning the services provided to customers while the 3rd party service provider plays the role of Quality Manager and Accounting Manager concerning the different basic service components he provides to the service retailer. Periodically, the Basic Service Component QoS Manager checks if the performance of its basic service components A and B respects the SLA signed with the service retailer. Indeed, it handles the deployment, the execution and the management of its service components. When it detects a "trouble", a 
"trouble ticket" is sent to the service retailer. The Basic Service Component Provider also exchanges with the service retailer the accounting data for the use of basic service components. These data are necessary to provide the billing function for the usage of the service the consumer does.

On the other hand, the service retailer achieves the "end-to-end" management of the service through the Service QoS Management function. It deploys, monitors and manages the different Component Integrator (CI). The CI represented in gray in figure 4 achieves the integration of basic service components $\mathrm{A}$ and $\mathrm{B}$. When it receives "trouble tickets" from the basic service component provider, it must be able to handle the problem at the service level. It implies that it must find out its services which are degraded and provides solutions. It may also send "trouble tickets" to the Management GUI of the service consumer. Besides, it can use basic service components provided by other 3rd party service providers in order to provide its customers with better Quality of Service.

\section{Implementation}

This section describes the prototype we are implementing to demonstrate the validity of our approach. Figure 5 represents the architecture of this prototype. This prototype is implemented over the NEC front-end middleware [2]. Like the Application Layer Active Networking proposal [8], the front-end middleware enables a good integration with existing IP networks by simply overlaying the basic network infrastructure. In fact, the front-end middleware enables the dynamic deployment of piece of code, called front-end, in active nodes located at the edge of the network. A front-end is devoted to the preliminary processing of data going from clients to servers. The use of the front-end concept brings different benefits: putting the front-ends at the edge nodes of the network reduces the service response time; front-ends provide customers with more user-friendly and customized interfaces; besides, application servers can subcontract specific tasks to these front-end elements and focus their resources on fundamental tasks; finally, front-ends limit the network traffic by only forwarding to servers the data they cannot process. It enables the network to provide some part of a business service, going beyond the "traditional" approaches like data caching or load balancing which only provides data.

This prototype can be considered as one possible implementation of the generic platform architecture detailed in the previous sections of this document. Actually, our prototype is composed of the following entities:

- The Service Client has the consumer business role. We assume these users access the network through Points of Presence, or POPs, and use the classical Web browser as service interface. Actually, the ubiquity of Web browsers makes them the de-facto standard for service interface;

- The Service Broker is playing the role of service retailer. Its function is to sell and manage business services to both individual and corporate customers. At a computational level, the function of Service Broker can be replicated over several nodes of the network;

- Finally, the Service Component Provider and the Content Provider play the business role of 3rd party service provider. The Service Component Provider provides the basic service components necessary to build up the service logic while the 


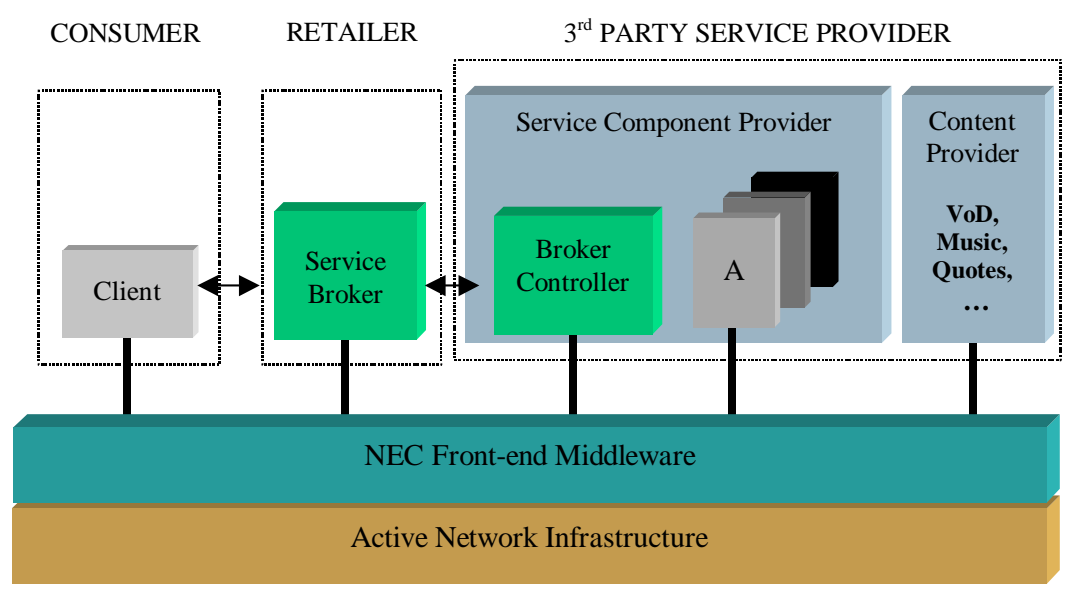

Figure 5. Overview of the Implementation Architecture.

Content Provider provides the content of the service. The business of the Service Component Provider is to develop and sell basic service components to service retailers. The Broker Controller deploys and manages the basic service components. Each 3rd party service provider has its own Broker Controller.

The broker business role defined in the TINA business model has not been implemented in our platform prototype. Actually, the number of actors of the telecom environment is currently limited and the retailer often also plays the role of broker at the same time. In a future work, we plan to separate these two business roles by implementing two separate entities. However, the front-end middleware has defined a White Page service that we use to implement our proposal. Besides, the use of the front-end middleware in our implementation enables the dynamic deployment of basic service components code within the network. In this context, the different entities of our proposal and the basic service components are implemented as front-end, enabling an on-demand and easy deployment of the service in the network. Besides, the authors are also investigating the Enterprise JavaBeans and CORBA Components technologies to improve our implementation.

\section{Conclusions}

This paper has described a platform architecture for deploying and managing component-based Internet services. In this context, a business service is viewed as an integration of basic service components. These components are reusable and shareable between business services. Our aim was to provide an enhancement to the concept of building blocks in order to provide service providers with platform for advanced Internet services management. The main philosophy of our generic platform architecture proposal is to enable service retailers to deploy a new service for immediate use by any consumer in the TINA system without standardizing the services with other retailers. Our platform architecture proposal makes the use of three strong and promising technologies: programmable networks, application components and workflow engine. 
The aim of this combination is to allow the dynamic deployment of component-based Internet services according to the requests of service consumers.

In the immediate future, we intend to complete the implementation of our prototype to check the validity of our approach. We also plan to define in more details the functional components of the Service Broker and Broker Controller entities. Finally, we want to make further research about the concepts of "service", "basic service component", and the degree of possible mapping of the IP service management architectures onto the Telecom standards and guidelines defined by the TINA-C and TM Forum.

\section{References}

1. Active Network Composable Services Working Group, "Composable Services for Active Networks", September 1998, http://www.cc.gatech.edu/projects/canes/compserv/cs-draft0-3.pdf

2. T. Egashira and Y. Kiriha, "Management Middleware for Application Front-ends on Active Networks", in Proceedings of NOMS 2000, Hawaii, April 2000.

3. Enterprise JavaBeans, http://java.sun.com/products/ejb/index.html

4. A. Juhola, I. Marshall, S. Covaci, T. Velte, M. Donohoe and S. Parkkila, "The Impact of Active Networks on Established Network Operators", in Proceedings of IWAN 1999, Berlin, July 1999.

5. G. Kar, A.Keller, S. Calo, "Managing Application Services over Service Provider Networks: Architecture and Dependency Analysis", in Proceedings of NOMS 2000, Hawaii, April 2000.

6. U. Legedza, D.J. Wetherall and J. Guttag, "Improving the Performance of Distributed Applications Using Active Networks”, IEEE Infocom, San Francisco, April 1998.

7. D. Lewis and al., "Implementing Integrated Management Business Processes uing Reusable Components", January 2000, tttp://www.cs.ucl.ac.uk/research/flowthru/content/finalrpt/flowthru-rpt.pdf

8. I. Marshall, M. Fry, L. Velasco and A. Ghosh, "Active Information Networks and XML”, in Proceedings of IWAN 1999, Berlin, July 1999.

9. OMG TC Document orbos/99-02-05, “CORBA Components: Joint Revised Submission", March 1, 1999.

10. Telecommunications Information Networking Architecture Consortium (TINA-C), “TINA Business Model and Reference Points - Version 4.0”, May 22, 1997.

11. TeleManagement Forum, "Generic Requirements for Telecommunications Management Building Blocks - GB 909 (part 1)", Evaluation Version 2.0, September 1999.

12. TeleManagement Forum, “Telecom Operations Map - GB 910”, Evaluation Version Release 1.0, October 1998.

13. D.L. Tennenhouse, at al., "A Survey of Active Network Research", IEEE Communications Magazine, Vol. 35, No. 1, January 1997, pp.80-85.

14. P. Wade and T. Richardson, "Workflow - A Unified Technology for Operational Support Systems", in Proceedings of NOMS 2000, Hawaii, April 2000. 